\section{Means to an end}

Growth-sustaining mutations that occur in human tumours can include the increased expression of telomerase, making inhibition of this enzyme a legitimate target for anticancer therapy. In their recent paper, Elizabeth Blackburn and colleagues demonstrate that the use of lentiviral-based vectors to disrupt telomerase function significantly inhibits tumour-cell growth.

Telomerase maintains the length of telomere repeats at chromosome ends through the concerted action of the catalytic protein subunit telomerase reverse transcriptase, and the RNA subunit telomerase RNA component (TERC). It is the short template domain of TERC that directs the synthesis of the telomeric DNA repeats, and previous experiments expressing mutant versions of this domain hinted at inhibition of tumour-cell growth. However, definitive conclusions were prevented by low expression levels. So, because of its efficient delivery and integration of genes into a wide range of cell types, including non-dividing cells, Blackburn and co-workers have used a safety-engineered lentiviral system to express a mutant form of the TERC template domain.

Expression of mutant TERC in telomerase-positive human melanoma and bladder cancer cell lines rapidly suppressed cell growth in vitro. However, in colon and breast tumour cell lines, which express high levels of endogenous TERC, mutant TERC was less effective. To overcome this problem, the authors designed a hairpin short interfering RNA (siRNA) to target a wild-typespecific sequence of TERC and co-expressed this and mutant TERC from the same lentiviral vector. Coexpression markedly increased the inhibition of proliferation compared with expression of either siRNA or mutant TERC alone.

So does mutant TERC expression have an effect in vivo? Human bladder cancer cells were infected with wild-type or mutant TERC, or were mock-infected, and then xenografted into the subrenal capsule of nude mice. Mock-infected and wild-type TERC-expressing cells established large and highly vascular tumours, whereas cells expressing mutant TERC showed reduced tumour growth and suppression of angiogenesis.

How does mutant-TERC expression inhibit proliferation? The authors show that this is neither dependent on telomere shortening nor tumour-suppressor p53 function. Evidence indicates that mutant TERC induces a DNA-damage response because of activation of the DNArepair-associated proteins GADD45 and WAF1, but this pathway requires further characterization.

In another approach, Blackburn and colleagues, in collaboration with Kashani-Sabet and colleagues, have used ribozymes - RNA molecules capable of sequence-specific cleavage of other RNA molecules - to target murine TERC in a mouse melanoma metastasis model. This time the plasmid-based ribozymes were delivered systemically using cationic liposomes, and melanoma metastases were significantly inhibited in treated mice compared with untreated controls. Together, the approaches used in both papers indicate the feasibility of targeting upregulated telomerase to treat a wide range of patients with cancer. Nicola McCarthy

\section{(1) References and links} ORIGINAL RESEARCH PAPERS Li, S. et al.

Rapid inhibition of cancer cell growth induced by lentiviral delivery and expression of mutanttemplate telomerase RNA and anti-telomerase short interfering RNA. Cancer Res. 64, 4833-4840 (2004) | Nosrati, M. et al. Antitumour activity of sytemically delivered ribozymes targeting murine telomerase RNA. Clin. Cancer Res. 19 4983-4990 (2004)

\section{WEB SITE}

Elizabeth Blackburn's lab:

http://biochemistry.ucsf.edu/ blackburn/

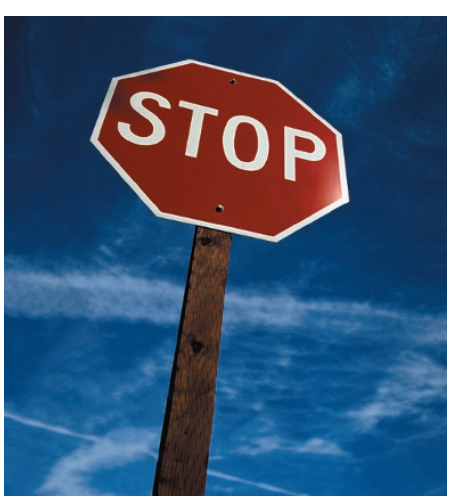

\section{IN BRIEF}

\section{TARGETED THERAPY}

Novel mode of action of c-kit tyrosine kinase inhibitors leading to NK cell-dependent antitumor effects.

Borg, C. et al. J. Clin. Invest. 114, 379-388 (2004)

Mutant KIT and platelet-derived growth factor (PDGF) receptors are the therapeutic targets of imatinib (Glivec) in gastrointestinal stromal tumours (GISTs). Laurence Zitvogel and colleagues have now shown that imatinib also acts on host dendritic cells to promote activation of natural killer cells, leading to antitumour effects. This explains how imatinib can be effective in GISTs that do not have mutant KIT and PDGF receptors.

\section{CANCER RISK}

\section{A major lung cancer susceptibility locus maps to} chromosome 6q23-25.

Bailey-Wilson, J. E. et al. Am. J. Hum. Genet. 75, 460-474 (2004)

Although the risk of lung cancer is greatly increased by cigarette smoking, genetic factors are also involved. To identify susceptibility genes, the authors conducted a genome-wide linkage analysis of 52 extended pedigrees. These were ascertained through probands with lung cancer who had several first-degree relatives with the disease. Using multipoint linkage analysis, the authors localized a major susceptibility locus to $6 \mathrm{q} 23-25$.

\section{TUMOUR SUPPRESSORS}

Chk1 is haploinsufficient for multiple functions critical to tumor suppression.

Lam, M. H. et al. Cancer Cell 6, 45-59 (2004)

CHK1 is an unusual tumour suppressor, as loss of a single copy is sufficient for tumorigenesis. Lam et al. showed that heterozygosity for Chkl in mouse mammary tissue contributes to tumorigenesis through inappropriate entry into $S$ phase, accumulation of DNA damage and inability to prevent premature mitosis. They also found that Chk1 is essential for the viability of adult cells, which might limit the use of anticancer agents inhibiting CHK1.

\section{CHEMOTOXICITY}

\section{Genome-wide discovery of loci influencing} chemotherapy cytotoxicity.

Watters, J. W. et al. Proc. Natl Acad. Sci. USA 101, 11809-11814 (2004)

Watters et al. carried out a genome-wide linkage study to identify regions involved in chemotherapy cytotoxicity. They uncovered two regions that influence the cellular effects of docetaxel and another that influences those of 5-fluorouracil. Identification of the causative genes in these regions could provide a first step towards individualized cancer chemotherapy. 